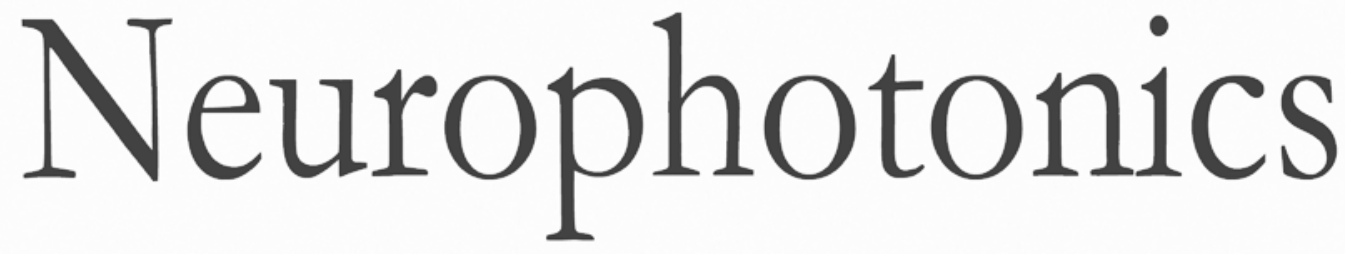

\title{
Integrated treatment modality of cathodal-transcranial direct current stimulation with peripheral sensory stimulation affords neuroprotection in a rat stroke model
}

\author{
Yu-Hang Liu \\ Su Jing Chan \\ Han-Chi Pan \\ Aishwarya Bandla \\ Nicolas K. K. King \\ Peter Tsun Hon Wong \\ You-Yin Chen \\ Wai Hoe $\mathrm{Ng}$ \\ Nitish V. Thakor \\ Lun-De Liao
}




\title{
Integrated treatment modality of cathodal-transcranial direct current stimulation with peripheral sensory stimulation affords neuroprotection in a rat stroke model
}

\author{
Yu-Hang Liu, ${ }^{\text {a,b }}$ Su Jing Chan, ${ }^{c}$ Han-Chi Pan, ${ }^{d}$ Aishwarya Bandla, ${ }^{a}$ Nicolas K. K. King,,${ }^{e, f}$ \\ Peter Tsun Hon Wong, ${ }^{g}$ You-Yin Chen, ${ }^{\mathrm{h}}$ Wai Hoe Ng, ${ }^{e, f}$ Nitish V. Thakor, ${ }^{\mathrm{a}, \mathrm{b}, \mathrm{i}}$ and Lun-De Liao ${ }^{\mathrm{a}, \mathrm{d}, \star}$ \\ ${ }^{a}$ National University of Singapore, Singapore Institute for Neurotechnology (SINAPSE), Singapore, Singapore \\ ${ }^{b}$ National University of Singapore, Department of Electrical and Computer Engineering, Singapore, Singapore \\ 'Massachusetts General Hospital and Harvard Medical School, Department of Radiology, Boston, Massachusetts, United States \\ ${ }^{\mathrm{d} N a t i o n a l ~ H e a l t h ~ R e s e a r c h ~ I n s t i t u t e s, ~ I n s t i t u t e ~ o f ~ B i o m e d i c a l ~ E n g i n e e r i n g ~ a n d ~ N a n o m e d i c i n e, ~ M i a o l i, ~ T a i w a n ~}$ \\ eNational Neuroscience Institute (NNI), Department of Neurosurgery, Singapore, Singapore \\ ${ }^{f}$ National Neuroscience Institute (NNI), SingHealth Duke-NUS Neuroscience Academic Clinical Program, Singapore, Singapore \\ 9National University of Singapore, Department of Pharmacology, Singapore, Singapore \\ ${ }^{\mathrm{h}}$ National Yang Ming University, Department of Biomedical Engineering, Taipei, Taiwan \\ 'Johns Hopkins University, Department of Biomedical Engineering, Baltimore, Maryland, United States
}

\begin{abstract}
Cathodal-transcranial direct current stimulation induces therapeutic effects in animal ischemia models by preventing the expansion of ischemic injury during the hyperacute phase of ischemia. However, its efficacy is limited by an accompanying decrease in cerebral blood flow. On the other hand, peripheral sensory stimulation can increase blood flow to specific brain areas resulting in rescue of neurovascular functions from ischemic damage. Therefore, the two modalities appear to complement each other to form an integrated treatment modality. Our results showed that hemodynamics was improved in a photothrombotic ischemia model, as cerebral blood volume and hemoglobin oxygen saturation $\left(\mathrm{SO}_{2}\right)$ recovered to $71 \%$ and $76 \%$ of the baseline values, respectively. Furthermore, neural activities, including somatosensory-evoked potentials ( $110 \%$ increase), the alpha-to-delta ratio $(27 \%$ increase), and the (delta + theta)/(alpha + beta) ratio ( $27 \%$ decrease), were also restored. Infarct volume was reduced by $50 \%$ with a 2 -fold preservation in the number of neurons and a 6 -fold reduction in the number of active microglia in the infarct region compared with the untreated group. Grip strength was also better preserved ( $28 \%$ higher) compared with the untreated group. Overall, this nonpharmacological, nonintrusive approach could be prospectively developed into a clinical treatment modality. ๑ 2017 Society of Photo-Optical Instrumentation Engineers (SPIE) [DOI: 10.1117/1.NPh.4.4.045002]
\end{abstract}

Keywords: transcranial direct current stimulation; peripheral sensory stimulation; electrocorticography; functional photoacoustic microscopy; neurovascular function; photothrombotic ischemia.

Paper 17105R received Jul. 7, 2017; accepted for publication Sep. 12, 2017; published online Oct. 4, 2017.

\section{Introduction}

Ischemic stroke, which is primarily caused by interruption of the blood supply in the brain by a thrombus, is a major cause of death and a leading cause of adult disability. ${ }^{1}$ Thrombolysis through the use of recombinant tissue plasminogen activator $(\mathrm{rtPA})^{2}$ is the only treatment for acute stroke currently approved by the U.S. Food and Drug Administration. However, it is only viable for $\sim 4 \%$ of stroke patients ${ }^{3}$ because it must be administered within $\sim 4 \mathrm{~h}$ of stroke onset. In addition, rtPA treatment may cause serious adverse effects, such as hemorrhagic conversion ${ }^{4}$ and bloodbrain barrier (BBB) disruption. ${ }^{5}$ On the other hand, neuroprotection is another widely researched therapeutic approach that aims to protect the brain from ischemic injuries and improve functional outcomes. ${ }^{1,6}$ Unfortunately, neuroprotective agents found to be effective in animal studies have not been proven effective in clinical trials. ${ }^{7}$ One possible reason may be the complex pathology 6 involved in stroke. Thus, it is advisable to widen the scope of research beyond pharmacotherapy to develop new types of therapeutic interventions. ${ }^{6,8-10}$
Transcranial direct current stimulation (tDCS) involves the application of a weak direct current transcranially to polarize target brain regions. ${ }^{11}$ In recent years, the neuroprotective effects of tDCS for clinical stroke recovery and treatment of hyperacute ischemia have been evaluated in animal models. ${ }^{12-14}$ In human studies, tDCS has been used to modulate brain activity in a number of neurological disorders including stroke and chronic pain. ${ }^{15-17}$ It has been reported that anodal tDCS (A-tDCS) increases cortical excitability, while cathodal tDCS (C-tDCS) leads to cortical inhibition. ${ }^{11,18}$ In addition, tDCS can be used to modulate hemodynamics, such as cerebral blood flow (CBF) and cortical neurotransmitters (e.g., gamma-aminobutyric acid and glutamate) by adjusting the polarity. ${ }^{14,19} \mathrm{C}$-tDCS applied immediately after the ischemic insult was reported to cause a $20 \%$ to $30 \%$ reduction in infarct volume when its protective effects in the hyperacute phase of stroke were evaluated, although the peri-ischemic inflammatory response was approximately the same as that in the control group (i.e., nonstimulated animals) at $72-\mathrm{h}$ postischemia. ${ }^{13,14}$ On the contrary, A-tDCS 
appeared to exacerbate dysregulation of the BBB and induce hemorrhage postischemia. These results thus indicate that tDCS may provide only modest protection against an ischemic insult. ${ }^{14}$ Interestingly, it has been shown that tDCS does not only modulate neuronal membrane potentials beneath the stimulus electrode, but also has a systematic effect on neuronal circuits remote from the stimulated site, likely covering most of the brain. ${ }^{20} \mathrm{We}$, however, have previously shown that peripheral sensory stimulation (PSS), applied through electrodes inserted into the forepaw contralateral to the cerebral ischemic insult, was able to increase blood volume to specific brain areas, resulting in rescue of cortical neurovascular functions from ischemic damage. ${ }^{21,22}$ Therefore, we proposed that a combination of tDCS and PSS may represent a more integrated nonpharmacological strategy for neuroprotection against cerebral ischemia. We further hypothesized that this integrated approach takes advantage of both types of stimulation to ameliorate exacerbation of injury (e.g., inhibition of cortical spreading depression) and to promote postischemic recovery (e.g., reperfusion). ${ }^{13,14,22}$ In this study, the proposed integrated intervention was evaluated to determine its potential beneficial effects in the hyperacute phase of ischemia. Electrocorticography-functional photoacoustic microscopy (ECoG-fPAM) was used to monitor changes in neurovascular functions, including somatosensory-evoked potentials (SSEPs), alpha-to-delta ratio (ADR), (delta + theta)/ (alpha + beta) ratio (DTABR), cerebral blood volume (CBV), and hemoglobin oxygen saturation $\left(\mathrm{SO}_{2}\right)$. Immunohistochemical microscopy and behavioral testing were employed to assess the neuroprotective effects.

\section{Methods}

\subsection{Electrocorticography-Functional Photoacoustic Microscopy System}

Figure 1 shows the experimental setup employed in this study, including the ECoG-fPAM system (i.e., the ECoG signal recording system with functional PA imaging), the photothrombotic

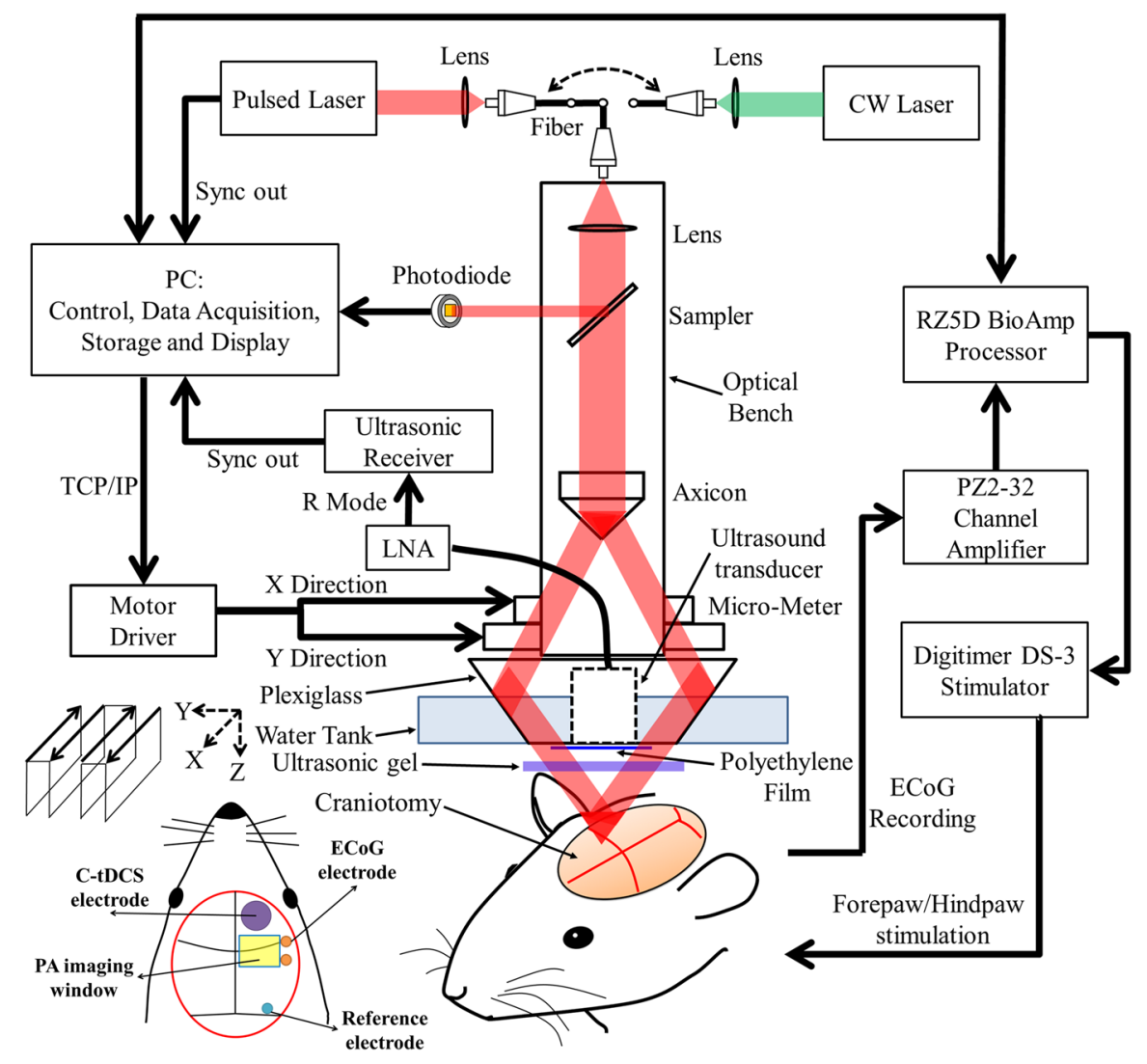

Fig. 1 Schematic diagram of the ECoG-fPAM system and the C-tDCS experimental setup. The ECoGfPAM system includes an ECoG signal recording system for neural activity evaluation and a functional PA imaging system for hemodynamic assessment. ECoG signals were acquired via two stainless steel epidural electrodes inserted into the S1FL region to evaluate evoked responses (i.e., SSEPs induced by forepaw stimulation) and resting-state neural activity. For the fPAM setup, $10-\mathrm{Hz}$ laser pulses were generated and coupled to the optical path of a dark-field microscope to illuminate the region of interest and to induce a PA effect. A customized 50-MHz transducer was used to detect the generated PA waves, which were then transferred to a computer for further data analysis. Ultrasonic gel was applied to the cortex for good coupling efficiency. B-scan PA images for $\mathrm{CBV} / \mathrm{SO}_{2}$ changes in the blood vessels of the ischemic region along the AP direction were acquired using the motorized scanning stage. To induce $\mathrm{PTI}$, a CW laser light was coupled to the optical path of the fPAM and was focused on the selected cerebral blood vessel within the PA imaging window. For C-tDCS intervention, the cathodal electrode was placed on the skull, while the anodal electrode was attached to the venter. For PSS intervention, forepaw/hindpaw stimulation was delivered via subdermal needle electrodes. 
ischemia (PTI) rat model, C-tDCS, and forepaw/hindpaw electrical stimulation (i.e., PSS). For ECoG recordings, three stainless steel epidural electrodes (including one reference electrode) were secured on the skull to acquire SSEPs and resting-state ECoG signals, which were recorded/processed via a commercial biosignal processor (RZ5D, Tucker-Davis Technologies, Florida). ${ }^{22}$ The trigger signals used to evoke neurovascular responses (e.g., functional hemodynamic changes) or induce PSS were also generated by this biosignal processor. The acoustic-resolution PAM system offers an axial resolution of $32 \mu \mathrm{m}$ and a lateral resolution of $61 \mu \mathrm{m}$, while the achievable penetration depth of the fPAM is estimated to be $3 \mathrm{~mm}$. A frequency-tripled Nd:YAG Q-switched laser (Surelite II-10, Continuum, California) was used to pump the optical parametric oscillator (Surelite OPO Plus, Continuum, California), generating 4-ns laser pulses at a pulse repetition rate of $10 \mathrm{~Hz}$. The pulsed laser light was delivered via a 1-mm multimodal fiber, which then passed through a collimation lens, an axicon, and a plexiglass mirror, forming a confocal dark-field illumination spot on the target. The laser exposure on the surface of the target was lower than the maximum permissible exposure limit of $20 \mathrm{~mJ} / \mathrm{cm}^{2}$ set by the American National Standards Institute. A custom-made $50-\mathrm{MHz}$ ultrasonic transducer (S-Sharp, Taiwan) was used to receive the laser pulse-induced PA signals. During the PA imaging process of in vivo experiments, the ultrasonic transducer was immersed in an acrylic water tank, while the hole at the bottom of the water tank was sealed with a piece of $15-\mu \mathrm{m}$ thick polyethylene film. A thin layer of ultrasonic gel was applied to the animal's cortex, and then the film was attached to the gel, ensuring good acoustic coupling of the generated PA signals. Afterward, the PA signals received by the ultrasonic transducer were preamplified by a low-noise amplifier (AU-3A-0110, Miteq Inc., New York) cascaded with an ultrasonic receiver (5073 PR, Olympus, Pennsylvania) and digitized by a computer-based 14-bit analog-to-digital (A/D) card (CompuScope 14200, GaGe, Illinois) at a 200-MHz sampling rate. Additionally, a photodiode (DET36A/M, Thorlabs, New Jersey) was used to monitor the fluctuations in laser light energy. The recorded signals from the photodiode were applied to compensate for variations in PA signals caused by the laser light energy instability.

\subsection{Animal Preparation}

Fifty-eight male Sprague Dawley rats weighing 250 to $300 \mathrm{~g}$ (InVivos Pte. Ltd., Singapore) were randomly divided into three groups for evaluation of neurovascular functions: control PTI only $(n=16)$ group, C-tDCS + PTI $(n=21)$, and C-tDCS + PSS + PTI $(n=21)$. Among them, 18 animals were equally divided into three groups and used for immunohistochemical studies. All experimental protocols were approved by the Institutional Animal Care and Use Committee of the National University of Singapore and the National Health Research Institutes of Taiwan. The animals were housed at a constant temperature and humidity with free access to food and water.

During the entire surgical and experimental procedures, the body temperature of the rats was maintained at $37^{\circ} \mathrm{C} \pm 0.5^{\circ} \mathrm{C}$ using a rectal probe (TCAT-2 Temperature Controller, New Jersey) and a self-regulating thermal plate. The rats were anesthetized with pentobarbital (i.e., $50 \mathrm{mg} / \mathrm{kg}$ bolus induction and $15 \mathrm{mg} / \mathrm{kg} / \mathrm{hr}$ maintenance, i.p. injections). The anesthetized rats were mounted on a custom-made acrylic stereotaxic head holder and placed on a bed pallet during the experiment.
A craniotomy was performed on the exposed skull with a high-speed drill (Micromotor drill, Stoelting, Illinois) to open a cranial window $\left(\sim 4 \times 4 \mathrm{~mm}^{2}\right)$ on the right side of the skull for B-scan PA imaging and PTI induction. Two stainless steel epidural electrodes were secured to the skull over the forelimb region of the primary somatosensory cortex (S1FL): +0.48 and $-0.92 \mathrm{~mm} \mathrm{AP},+4.5 \mathrm{~mm} \mathrm{ML}$ for ECoG recording. The reference electrode was positioned $3 \mathrm{~mm}$ lateral to the lambda, as shown in Fig. 1. These electrodes were connected via silver wires to the data acquisition system (RZ5D) through a connector (ZIF-Clip headstage, Tucker-Davis Technologies, Florida). For subsequent procedures, the distance between the interaural line and the bregma was used to position the head in the PPAM system.

\subsection{In Vivo Rat Photothrombotic Ischemia Model}

The in vivo PTI model was established according to Watson et al. ${ }^{23}$ Focal ischemia was induced by blocking a selected cortical blood vessel [i.e., a distal branch of the middle cerebral artery (MCA)] through the cranial window over the S1FL region of the right hemisphere. ${ }^{22}$ This effect was achieved by first injecting the photosensitizer Rose Bengal (Sigma, Missouri) diluted to $10 \mathrm{mg} / \mathrm{ml}$ in HEPES-buffered saline over the course of $2 \mathrm{~min}$ into the tail vein at $0.2 \mathrm{ml} / 100 \mathrm{~g}$ rat body weight. The selected cortical blood vessel was then illuminated using a $5 \mathrm{~mW}, 532 \mathrm{~nm}$ CW laser light (AMGA-005, ONSET, Taiwan) to generate an occlusion. ${ }^{23,24}$ The $\mathrm{CW}$ laser light was coupled to the dark-field optical path of the ECoG-fPAM system and focused on the selected cortical blood vessel for at least 15 min until a stable clot was formed. ${ }^{22,25}$

\subsection{Neural Activity and Hemodynamics Assessments}

As shown in Fig. 2(a), 60-s resting-state ECoG signals were recorded from the S1FL region of the right hemisphere to evaluate the effect of ischemia on neural activity, without an explicit stimulus applied to the rat. To evaluate functional changes in stimulus-evoked SSEPs and $\mathrm{CBV} / \mathrm{SO}_{2}$, forepaw electrical stimulation was delivered as a trigger via subdermal needle electrodes inserted into the left forepaw of the rat (i.e., contralateral to the ischemic hemisphere). ${ }^{26,27}$ The electrical stimulation was delivered using a current stimulator (DS3, Digitimer, Hertfordshire, England). To synchronize this stimulator with the multichannel RZ5D biosignal processor, trigger pulses for controlling the stimulator were delivered via an output port of the processor. After the resting-state ECoG was recorded, a 5-s monophasic constant current with a 2-mA intensity and a $0.2-\mathrm{ms}$ pulse width at a frequency of $3 \mathrm{~Hz}$ was delivered to induce SSEPs corresponding to neural activity.

On the other hand, for assessing functional changes in different locations, three B-scan PA images were acquired during each hemodynamics assessment block. Thus, for evoking hemodynamic responses in these planes of the cranial window (i.e., ML: $+3.2,+3.7$, and $+4.2 \mathrm{~mm}$ ), three 1-s electrical stimulations were delivered, followed by $30 \mathrm{~s}$ of PA B-scan imaging at 808 and $880 \mathrm{~nm}$ wavelengths $\left(\lambda_{808}\right.$ and $\left.\lambda_{880}\right)$ for the selected cortical blood vessels in the S1FL region of the right hemisphere [Fig. 2(a)]. The time points of neural activity and hemodynamics assessments during the experiments are shown in Fig. 2(b) with blue and green arrows, respectively. 
(a) Neural activity

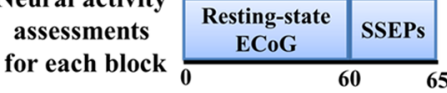

Time (second)

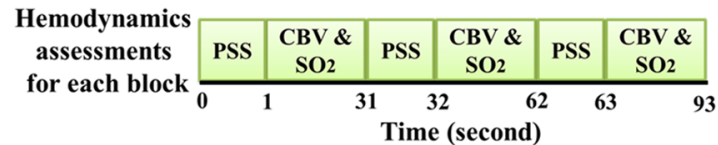

(b) Neurovascular

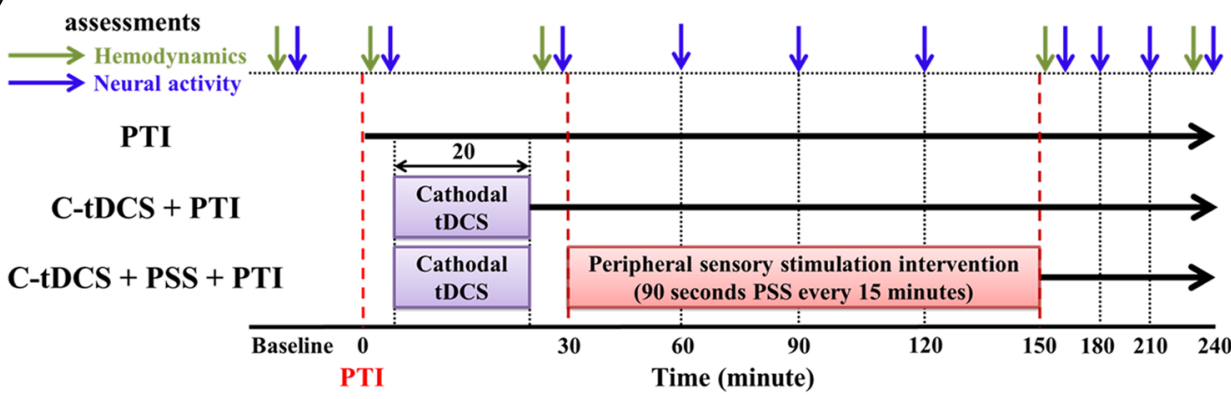

Fig. 2 Schematic diagram of the assessment and the therapeutic intervention protocols. (a) To evaluate neural activity, in each block, 60 -s resting-state ECoG signals were recorded from the S1FL region of the right hemisphere, followed by $5 \mathrm{~s}$ monophasic constant current stimulation at $3 \mathrm{~Hz}$ to induce SSEPs of neural activity. To assess functional changes in the three different locations, three B-scan PA images were acquired in each hemodynamics assessment block. Each 1-s electrical stimulation was delivered and followed by $30 \mathrm{~s}$ of PA B-scan imaging at $\lambda=808$ or $\lambda=880$ for the selected cortical blood vessel. (b) Neurovascular functions were evaluated using the ECoG-fPAM system in all three groups (PTI, C-tDCS + PTI, and C-tDCS + PSS + PTI). The blue and green arrows indicate the time points of neural activity and hemodynamic assessments, respectively. The purple block shows the duration of C-tDCS administration. Current stimulation at $2 \mathrm{~mA}$ and $3 \mathrm{~Hz}$ with a 0.2-ms pulse width was delivered for $90 \mathrm{~s}$ every 15 min during the PSS intervention session (red block).

\subsection{Experimental Protocols, Cathodal-Transcranial Direct Current Stimulation, Peripheral Sensory Stimulation, and Integrated Therapeutic Intervention}

Before ischemia induction, neurovascular functions were evaluated via the ECoG-fPAM system in all three groups (PTI, C-tDCS + PTI, and C-tDCS + PSS + PTI), as shown in Fig. 2(b). C-tDCS was applied using a current stimulator (Model 2100, A-M systems, Washington) to deliver a constant current of $400 \mu \mathrm{A}$ for $20 \mathrm{~min}$ immediately after PTI. Previous studies have shown that $\mathrm{C}$-tDCS has a protective effect against ischemia when applied in the very early stage. ${ }^{13,14}$ Therefore, the duration of 20 min was selected accordingly, ${ }^{12,14,18,20}$ and it appeared to be optimal in our preliminary studies. The cathodal electrode was attached transcranially to the rat at an anatomical location 5-mm anterior and 3-mm lateral to the bregma (Fig. 1), while the anodal electrode was attached to the venter and surrounded by gauze to avoid displacement. The contact area of the cathodal electrode was $0.196 \mathrm{~cm}^{2}$, whereas the area of the anodal electrode was $4 \times 3.5 \mathrm{~cm}^{2}$. The current intensity of $400 \mu \mathrm{A}$, corresponding to a current density of $20.37 \mathrm{~A} / \mathrm{m}^{2}$, was within the range of intensities used in other acute experiments on animals. ${ }^{14}$ For PSS, a total of four subdermal needle electrodes (two for forepaw and two for hindpaw stimulation) were inserted into the rat to deliver the PSS. Current stimulation of $3 \mathrm{~Hz}$ with a $0.2-\mathrm{ms}$ pulse width and $2 \mathrm{~mA}$ was delivered for $90 \mathrm{~s}$ (i.e., 270 corresponding sweeps) every $15 \mathrm{~min}$ for $2 \mathrm{~h}$ after the 20-min C-tDCS [Fig. 2(b)].

\subsection{Data Analysis of the Cerebral Blood Volume and Hemoglobin Oxygen Saturation Measurements}

Relative functional changes in $\mathrm{CBV}$ and $\mathrm{SO}_{2}$ were monitored/ evaluated via two optimized wavelengths (i.e., $\lambda_{808}$ and $\lambda_{880}$, respectively). ${ }^{26}$ It was assumed that $\mathrm{CBV}$ is proportional to the cross section of a single vessel at 808 -nm wavelength $\left(\lambda_{808}\right){ }^{28}$ Based on the PA B-scan image at $\lambda_{808}\left[I_{R(808)}\right]$ in the selected plane of the S1FL region, the cross-sectional area $\left\{A\left[I_{R(808)}\right]\right\}$ of the selected blood vessel was calculated using the total blood vessel pixel count, while a blood vessel pixel was defined as a pixel that possessed a PA signal three times greater than the background signal. ${ }^{21-23}$ Then, functional CBV changes $\left(R_{\mathrm{CBV}}\right)$ were derived using the following equation:

$R_{\mathrm{CBV}}(t)=\frac{A\left[I_{R(808)}(t)\right]}{A\left[I_{R(808), \text { baseline }}\right]}$,

where $t$ is the time in each block, $A\left[I_{R(808)}(t)\right]$ represents the cross-sectional area at a given time in each block, and $A\left[I_{R(808) \text {,baseline }]}\right]$ is the baseline of the cross-sectional area estimated from the baseline image acquired immediately before the onset of stimulation in the block. ${ }^{21}$ Thus, relative functional $\mathrm{CBV}$ changes $\left(R_{\mathrm{CBV}}\right)$ in a single vessel could be monitored by assessing the changes in the cross-sectional area of the selected vessel compared with the baseline value.

PA signal changes at $880 \mathrm{~nm}$ wavelength $\left(\lambda_{880}\right)$ were sensitive to $\mathrm{SO}_{2}$ changes because larger absorption changes in the near-infrared spectrum occurred at approximately $\lambda_{880}$ when the $\mathrm{SO}_{2}$ levels changed. Images of functional $\mathrm{SO}_{2}$ changes $\left[\Delta I_{F(880)}(\mathrm{t})\right]$ at a given time point $t$ in each block were assessed using the following equation:

$$
\begin{aligned}
\Delta I_{F(880)}(t) & =\frac{I_{(880)}(t)}{I_{R(808)}(t)}-\frac{I_{(880), \text { baseline }}}{I_{R(808), \text { baseline }}} \\
& =I_{F(880)}(t)-I_{F(880), \text { baseline }},
\end{aligned}
$$

where $I_{(880)}$ (i.e., the PA image acquired at $\lambda_{880}$ ) is normalized to $I_{R(808)}$ on a pixel-by-pixel basis and $I_{(880) \text {,baseline }}$ is the baseline image at $\lambda_{880}$ acquired immediately before the onset of stimulation in each block. $^{26}$ Note that negative values of 
$\Delta I_{F(880)}$ [i.e., a positive $-\Delta I_{F(880)}$ ] indicate increases in $\mathrm{SO}_{2}$ levels and vice versa. ${ }^{26}$ The mean functional $\mathrm{SO}_{2}$ changes $\left[R_{\mathrm{SO}_{2}}(t)\right]$ in a single blood vessel during the stimulation period were determined as follows:

$$
\begin{aligned}
R_{\mathrm{SO}_{2}}(t)= & \sum_{(X, Z \in \text { vessel pixel })} \frac{I_{F(880)}(x, z, t)}{A\left[I_{R(808)}(t)\right]} \\
& -\sum_{(X, Z \in \text { vessel pixel })} \frac{I_{F(880), \text { baseline }}(x, z, t)}{A\left[I_{R(808), \text { baseline }}(t)\right]} .
\end{aligned}
$$

From Eqs. (2) and (3), the relative changes in $\mathrm{SO}_{2}\left(R_{\mathrm{SO}_{2}}\right)$ could also be independently determined using the fPAM system. As $R_{\mathrm{CBV}}$, the relative changes in $\mathrm{SO}_{2}$ were calculated compared with their baseline values (which were recorded immediately before stimulation) over the selected region. On the other hand, the $R_{\mathrm{CBV}}$ and $R_{\mathrm{SO}_{2}}$ values recorded before ischemia onset for each animal were regarded as the normal state (baseline) of $\mathrm{CBV}$ and $\mathrm{SO}_{2}$ function, which were compared with the $R_{\mathrm{CBV}}$ and $R_{\mathrm{SO}_{2}}$ values post-PTI for evaluating the effect of the interventions. Additional details on the data analysis of functional changes in $\mathrm{CBV}$ and $\mathrm{SO}_{2}$ in specific regions are available in our previous studies. ${ }^{21,29}$

\subsection{Data Analysis of Electrocorticography Recording}

SSEP measurements can potentially predict functional recovery after stroke, as SSEPs correlate well with the level of disability ${ }^{30}$ therefore, SSEPs have been used as an objective way to assess the integrity of sensory and motor pathways of the central nervous system and were used as a marker of neural activity in this study. ${ }^{31}$ Changes in the peak-to-peak amplitude (PPA) between P1 (i.e., the first positive peak after stimulation) and $\mathrm{N} 1$ (i.e., the first negative peak directly following P1) were evaluated, as these values can indicate the presence of cortical dysfunction. ${ }^{31-33}$ In this way, SSEPs were quantitatively evaluated and used to assess neural activity. The sampling rate for recording ECoG signals was $1 \mathrm{kHz}$. The recorded signals were pre-amplified through the PZ2-32 preamplifier and bandpass filtered between 0.3 and $150 \mathrm{~Hz}$ via an RZ5D processor. The SSEP signals evoked by left forepaw electrical stimulation were recorded pre and postischemia in the S1FL region of the right hemisphere. As mentioned in the previous section, the trigger pulses for controlling the current stimulator were generated by the biosignal processor, along with synchronous recording timestamps of each stimulus pulse onset and SSEP sweep. We administered $3 \mathrm{~Hz}$ stimulation for $5 \mathrm{~s}$ to evaluate SSEPs, and a total of 15 corresponding sweeps were extracted and averaged to generate SSEP waveforms over a 100 -ms epoch poststimulus pulse. Afterward, the P1 and N1 values were extracted from the recorded SSEP waveforms to further calculate the PPA based on these two components. The percentage change in SSEPs was computed relative to the baseline value for both channels in which the baseline value was recorded before the onset of PTI for each animal and was regarded as the normal neural activity status. In this study, two-channel SSEPs from the electrodes were averaged to represent the state of neural activity at each time point, as shown in Fig. 2 (blue arrows).

Delta (i.e., 0.3 to $3 \mathrm{~Hz}$ ) and alpha (i.e., 8 to $13 \mathrm{~Hz}$ ) frequency bands have been reported to be strong indicators of both injury and recovery after ischemia. ${ }^{34}$ Thus, the ADR [i.e., the ratio between alpha power and delta power (alpha/delta)] was used in this study to monitor functional neural changes of resting- state ECoG signals pre- and post-PTI; ${ }^{35}$ a higher ratio value indicates a greater degree of neural activation, and vice versa. On the other hand, theta (i.e., 4 to $7 \mathrm{~Hz}$ ) and beta (i.e., 14 to $20 \mathrm{~Hz}$ ) activity may also be altered in stroke. ${ }^{36-38}$ In comparison to the ADR, the DTABR [(delta + theta $) /($ alpha + beta $)$ ratio] additionally includes both the theta and beta bands to predict spontaneous neurological improvement after stroke. ${ }^{39,40}$ Fast Fourier transform was used to calculate the mean power over the two channels in all frequency bands. ${ }^{35}$ The percentage changes in the ADR and the DTABR were calculated with respect to the baseline value for both channels in which the baseline value was recorded before the onset of PTI and was regarded as the normal neural activity status. The two-channel $\mathrm{ADR}$ and DTABR values at the ischemic region were averaged to acquire a mean value representing the state of each evaluating block. More information on the detailed calculations of SSEPs, ADR, and DTABR are available in our previous studies. ${ }^{22,25,39,40}$

\subsection{Immunohistochemistry}

Rats were overdosed with pentobarbital $(300 \mathrm{mg} / \mathrm{ml})$ and perfused with $0.1 \mathrm{~mol} / \mathrm{L}$ phosphate buffer through the heart. The brains were then removed and snap frozen in crushed dry ice. Coronal sections $(30 \mu \mathrm{m})$ in the regions of interests $(+1,+0.7$, $+0.4,+0.1,-0.2,-0.5$, and $-0.8 \mathrm{~mm}$ ) were cut using a cryostat and mounted on glass slides. Antibodies against NeuN (1:200, Millipore, Massachusetts) and ED-1 (1:200, Millipore, Massachusetts) were used, and slides were incubated at $4^{\circ} \mathrm{C}$ overnight. Sections were then washed and incubated with Alexa Fluor 555-conjugated goat antimouse (Thermo Fisher Scientific, Massachusetts) secondary antibody for $1 \mathrm{~h}$ at room temperature. Brain slices were counterstained by 4',6-diamidino-2-phenylindole (Thermo Fisher Scientific, Massachusetts) and finally mounted with ProLong Gold (Thermo Fisher Scientific, Massachusetts) mounting media. As a negative control, sections were probed with phosphate-buffered saline (PBS) with $0.1 \%$ Triton instead of antibodies overnight. All fluorescent images were captured with an Olympus IX71 (Olympus, Japan) laser scanning confocal microscope, and images captured were processed by Image-Pro Insight software (Media Cybernetics, Maryland). Cell counts were performed by quantifying the number of immunopositive cells in a selected $1 \mathrm{~mm}^{2}$ site at the infarcted region in a blinded manner.

\subsection{Measurement of the Infarct Volume and Behavioral Assessment}

The infarct volume was measured using 2,3,5-triphenyl-tetrazolium chloride (TTC). ${ }^{41}$ At $48 \mathrm{~h}$ following the onset of PTI, the rats were overdosed with pentobarbital $(300 \mathrm{mg} / \mathrm{ml})$, and the brains were rapidly removed, washed in $1 \times$ PBS at room temperature, and snap frozen in dry ice until use. Six serial $1.5-\mathrm{mm}$ coronal sections were cut from each brain (from 4-mm anterior to 5-mm posterior to the bregma). The sections were stained with 2\% TTC (chloride, T84859, Sigma, Missouri) for $12 \mathrm{~min}$ at $37^{\circ} \mathrm{C}$ in the dark, followed by overnight immersion in $4 \%$ paraformaldehyde in $0.1 \mathrm{~mol} / \mathrm{L} \mathrm{PBS}, \mathrm{pH} 7.4$, at $4^{\circ} \mathrm{C}$. The infarcted tissue remained unstained (i.e., white), whereas normal tissue was stained red. The volume of the ischemic infarct was traced and calculated using ImageJ software (NIH Image). The infarct volume was calculated by adding the infarct areas of all sections and multiplying by the slice thickness. The true infarct volume was obtained by correction for brain edema as follows: ${ }^{22}$ 
Percentage of corrected infarct volume $=$

$$
\left\{\frac{[\text { Total lesion volume }-(\text { nonischemic hemisphere volume }- \text { ischemic hemisphere volume })]}{\text { Ischemic hemisphere volume }}\right\} \times 100 \text {. }
$$

Wood et al. ${ }^{42}$ demonstrated that photothrombotic cortical lesions produce a deficit in grip strength, which could be observed at $24 \mathrm{~h}$ post-PTI. For behavioral assessment, the grip strength test was conducted in a blinded manner 2 days post-PTI. A total of five grip strength trials were performed for each animal in $5 \mathrm{~min}$ intervals. Each trial included three pulls, and the average of all 15 pulls was used for data analysis.

\subsection{Statistical Analysis}

Baseline values of $\mathrm{CBV}, \mathrm{SO}_{2}$, SSEPs, ADR, and DTABR were used to normalize the observed post-PTI changes (i.e., the most extreme value post-PTI was scaled to 0 , and the baseline value was scaled to 1). The changes in SSEPs, ADR, and DTABR in the ischemic hemisphere between cases were assessed using repeated-measures analysis of variance (ANOVA) with Fisher's least significant difference (LSD) posthoc analysis. ${ }^{43}$ Caseto-case differences in averaged PA signals of the studied regions and changes in cross-sectional areas (i.e., $\mathrm{CBV}$ and $\mathrm{SO}_{2}$ changes) were examined using paired $t$-tests (i.e., $p<0.05$, $n=40$ ). The significance of the relative changes observed in the averaged PA signals of the studied areas in response to electrical stimulation was analyzed using the Wilcoxon matchedpairs signed-rank test (i.e., two-tailed, $p<0.05, n=40$ ). ${ }^{26}$ Immunofluorescence and behavioral data were assessed by a one-way ANOVA followed by a posthoc (Bonferroni) test (i.e., $p<0.05, n=6$ ). All statistical analyses were performed using SPSS (version 10.0, SPSS ${ }^{\circledR}$ ). Data are presented as the mean \pm standard deviation (S.D.). Statistical significance was reached for $p<0.05$.

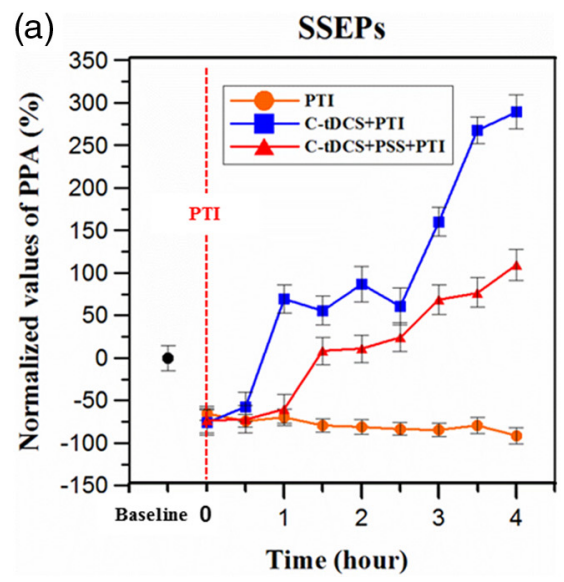

\section{Results}

\subsection{Effect of Interventions on Neural Activity During Hyperacute Phase of Photothrombotic Ischemia}

Figure 3 shows the PPA of SSEP waveforms and the ADR and DTABR of resting-state ECoG signal recordings pre- and post-PTI, which were used to indicate neural activity in this study. ${ }^{33,35,40}$ As shown in Fig. 3(a), the PPA of all three groups (i.e., PTI, C-tDCS + PTI, and C-tDCS + PSS + PTI) decreased by $\sim 70 \%$ from the baseline value immediately after the induction of PTI $(p<0.05)$. There was no spontaneous recovery during the hyperacute phase (within $4 \mathrm{~h}$ ) as observed in the PTI-only group. In contrast, C-tDCS treatment caused the PPA to increase, reaching $\sim 290 \%$ above the baseline level within $4 \mathrm{~h}(p<0.05)$. The combined treatment of C-tDCS + PSS yielded similar patterns of change except that the increase was more modest, reaching only approximately $100 \%$ above the baseline level $(p<0.05)$. The observed changes in ADR were similar to those of PPA [Fig. 3(b)], recovering from a decreased level of $\sim 40 \%$ of baseline level to $\sim 140 \%$ and $30 \%$ above baseline in the C-tDCS-treated and C-tDCS + PSS treated groups, respectively, within 4-h post-PTI.

DTABR, unlike PPA and ADR, increased to $\sim 100 \%$ above the baseline value in all three groups [Fig. 3(c)] $(p<0.05)$. Here, both treatment groups showed similar changes and DTABR returned to baseline within $1 \mathrm{~h}$. Over the next $3 \mathrm{~h}$, DTABR decreased to $\sim 50 \%$ and $30 \%$ of the baseline value for the C-tDCS and C-tDCS + PSS groups, respectively $(p<0.05)$, but, overall, the two treatment groups did not
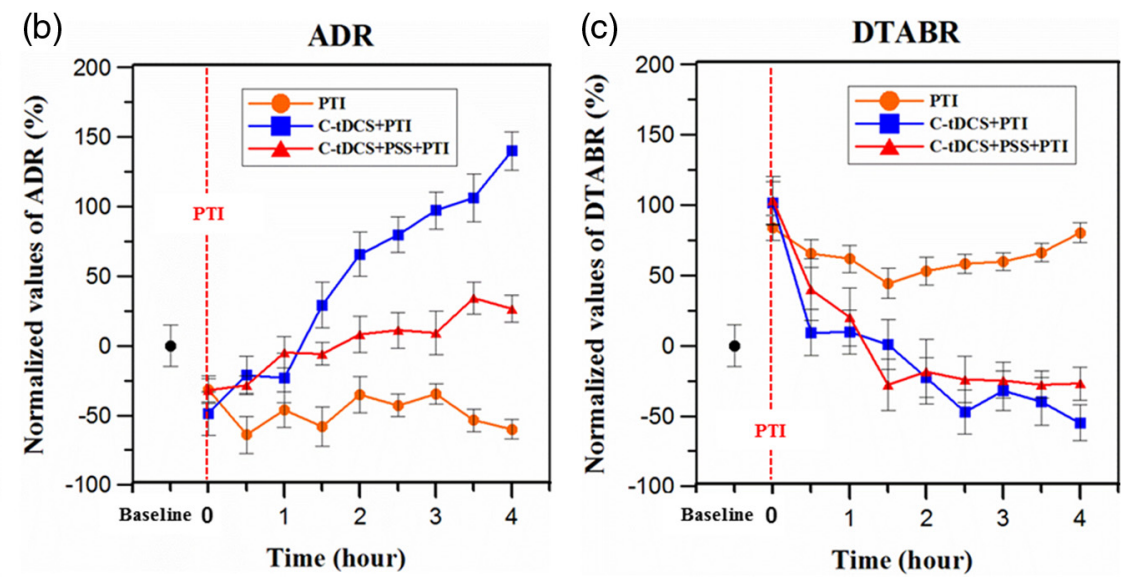

Fig. 3 Effect of interventions on neural activity during the hyperacute phase of PTI. (a) Changes in the normalized PPA of SSEPs are shown. (b and $c$ ) The ADR and DTABR values of resting-state ECoG signal recordings were also used as indicators to evaluate neural functions pre- and post-PTI in this study. Both ADR and DTABR significantly recovered and exceeded the baseline values for the C-tDCS + PTI and C-tDCS + PSS + PTI groups, while the values of the PTI group worsened, consistent with the PPA results. The results showed that therapeutic interventions including C-tDCS were able to restore neural activity in the ischemic region during the hyperacute phase of PTI. The values of all three indicators for each time point were averaged over the two electrode channels and plotted with standard deviations, as these two channels were both located in the S1FL region and within the ischemic area. These results represented the overall state of each evaluation block and were used to compare the values obtained at various time points. A posthoc analysis comparing the indicators at each time point was also performed. 
significantly differ. Again, no recovery of DTABR was observed in the PTI-only group. The above results showed that C-tDCS treatment was able to restore neural activity in the ischemic region during the hyperacute phase of PTI. Moreover, PSS modulated the effects of C-tDCS toward baseline levels for at least two of the three parameters measured.

\subsection{Effect of Interventions on Hemodynamics During the Hyperacute Phase of Photothrombotic Ischemia}

PA imaging was employed to evaluate the effect of interventions on hemodynamics as measured by $\mathrm{CBV}$ and $\mathrm{SO}_{2}$ following PTI.
To assess different locations near the ischemic induction region, three planes of the cranial window were scanned to evaluate the hemodynamic changes at the PTI induction region $(+3.7 \mathrm{~mm}$ from the bregma) and its downstream $(+3.2 \mathrm{~mm})$ and upstream $(+4.2 \mathrm{~mm})$ regions, as shown in Fig. 4(a). The PA-measured CBV significantly changed in the C-tDCS + PSS + PTI group at different locations pre- and post-PTI [Fig. 4(b)]. The results showed that the $\mathrm{CBV}$ of the PTI induction region significantly recovered to $\sim 50 \%$ of the baseline value during the hyperacute phase of ischemia following C-tDCS + PSS treatment $(p<0.05)$. On the other hand, the B-scan images of upstream and downstream regions also showed similar recovery of CBV after applying the intervention. (a)

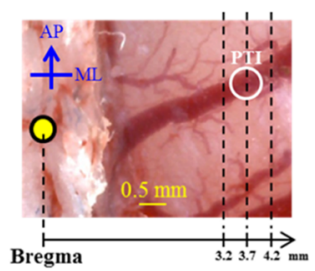

(b)

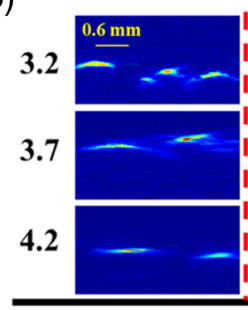

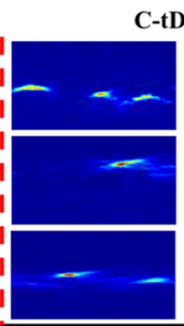

-tDCS + PSS + PTI group

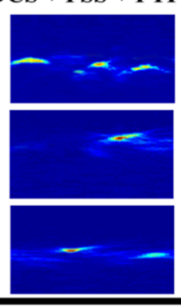

30

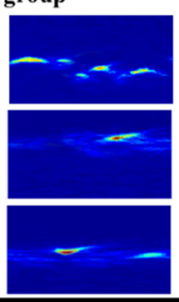

150

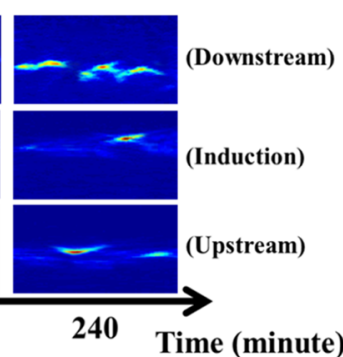

240 Time (minute)

(c)
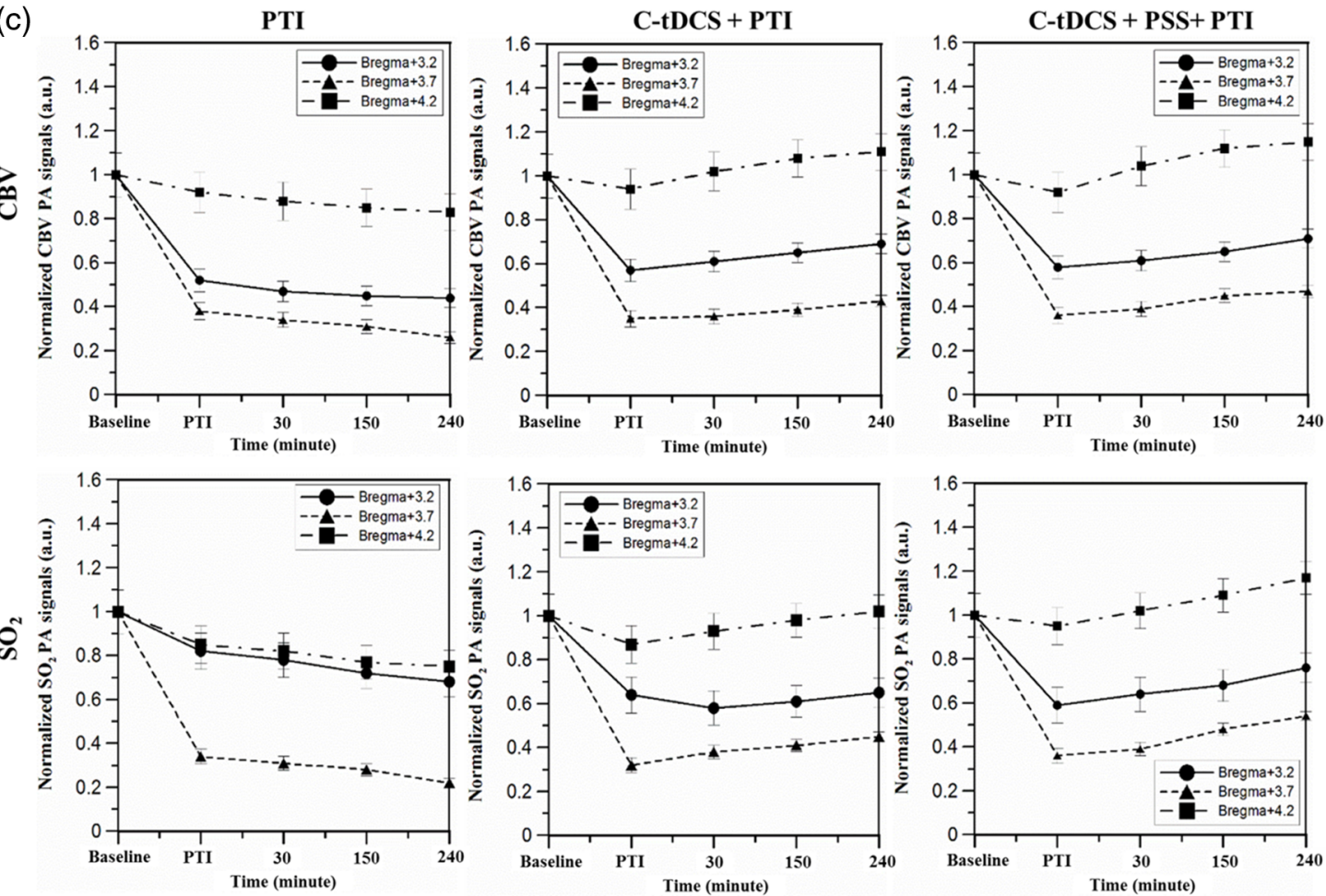

Fig. 4 Changes in the normalized $\mathrm{CBV}$ and $\mathrm{SO}_{2}$ values pre- and postischemia. (a) To assess different locations near the ischemic induction region, three planes of the cranial window (i.e., ML: $+3.7 \mathrm{~mm}$, $+3.2 \mathrm{~mm}$, and $+4.2 \mathrm{~mm}$ ) were scanned to evaluate the hemodynamic changes at the PTI induction region and its downstream and upstream regions, respectively. Note that the photo shown here is only for illustration [not related with PA signals in (b)]. (b) The PA-measured CBV $\left(R_{\mathrm{CBV}}\right)$ changes of the C-tDCS + PSS + PTI group at three locations pre- and post-PTI. The results showed that CBV of the PTI induction region could not be recovered to the baseline value, which might be because this region had become the ischemic core due to C-tDCS (decreasing the CBF over the stimulated region). On the other hand, the B-scan images of downstream and upstream regions showed that more $\mathrm{CBV}$ was restored to the surrounding regions (i.e., reperfusion), which may protect the ischemic area. (c) The normalized CBV $\left(R_{\mathrm{CBV}}\right)$ and $\mathrm{SO}_{2}\left(-R_{\mathrm{SO}_{2}}\right)$ changes of all groups at three locations. The results indicated that C-tDCS + PSS intervention led to the greatest improvement in ischemic outcome according to hemodynamics $(p<0.05)$. 
The normalized $\mathrm{CBV}\left(R_{\mathrm{CBV}}\right)$ and $\mathrm{SO}_{2}\left(-R_{\mathrm{SO}_{2}}\right)$ changes of the three groups at the three different locations over time are shown in Fig. 4(c). Following PTI induction, the relative $\mathrm{SO}_{2}$ of all three groups decreased initially by $\sim 66 \%$ relative to baseline at the PTI induction region. Both treatment groups recovered to $\sim 50 \%$ of the baseline value after $240 \mathrm{~min}$, while the untreated group further deteriorated to $80 \%$ below baseline. On the other hand, the results indicated that the CBV value of the C-tDCS + PSS + PTI group was the highest among all groups $4 \mathrm{~h}$ postischemia at the three B-scan locations. The CBV at the PTI induction region initially decreased significantly to $\sim 36 \%$ of the baseline level after PTI induction in all three groups $(p<0.05)$. This was followed by a significant recovery to $47 \%$ of the baseline value in the C-tDCS + PSStreated group at $240 \mathrm{~min}(p<0.05)$. In the upstream and downstream regions, the initial decreases in $\mathrm{SO}_{2}$ and $\mathrm{CBV}$ following PTI were markedly less than those at the induction region. However, consistent observations were made in which both $\mathrm{SO}_{2}$ and $\mathrm{CBV}$ recovered after the initial decreases in both $\mathrm{C}$-tDCS-treated groups but not in the untreated group. In summary, the results demonstrated that the C-tDCS + PSS intervention resulted in substantial improvement in stroke outcome during the hyperacute phase of ischemia. We also noted that the downstream $\mathrm{SO}_{2}$ levels were higher in the untreated (a) $=$ C-tDCS + PSS + PTI $\because$ C-tDCS + PTI $\because$ PTI

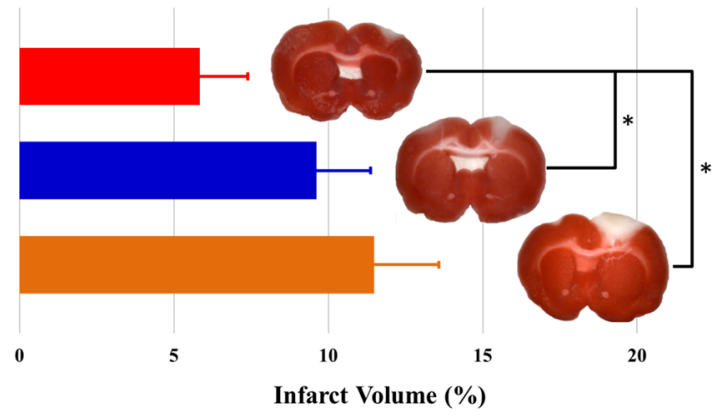

(b)

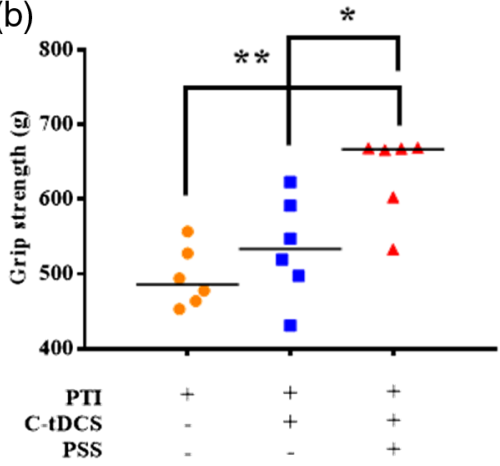

Fig. 5 C-tDCS + PSS protected against ischemic injuries in vivo. (a) Infarct volume was significantly reduced by C-tDCS + PSS compared with no treatment (PTI alone), while C-tDCS was not effective. Representative TTC-stained coronal brain sections show infarct areas (white) on the right. The lesion volumes are expressed as a percentage of the ischemic hemispheric volume after correction for edema. (b) Functional recovery was assessed by measuring the grip strength. Similarly, grip strength was significantly preserved in rats treated with C-tDCS + PSS compared with animals with no treatment or C-tDCS treatment. ${ }^{*} p<0.05$ and ${ }^{* *} p<0.01$.

(a)

PTI

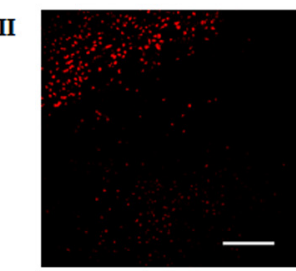

I

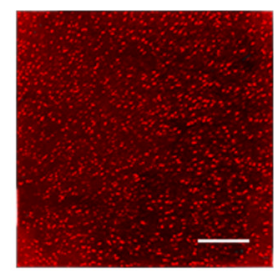

PTI + C-tDCS
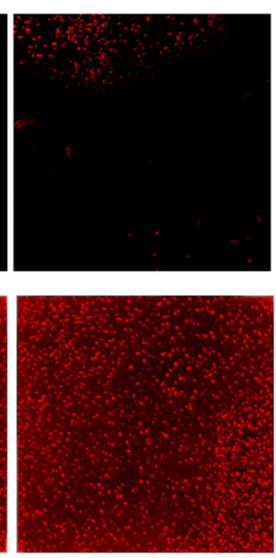

PTI + C-tDCS + PSS
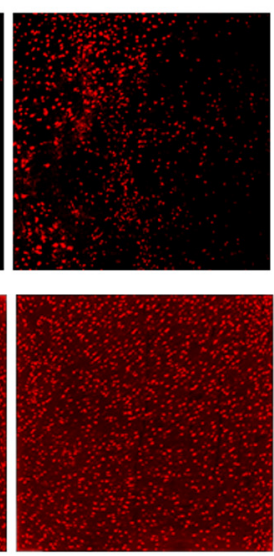

(b)

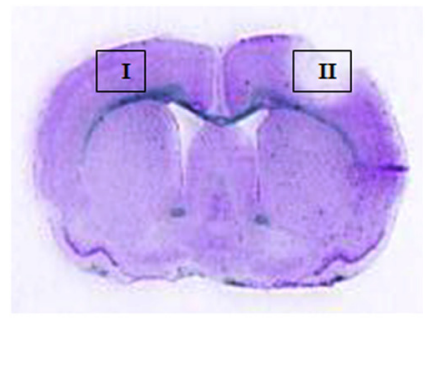

(c)

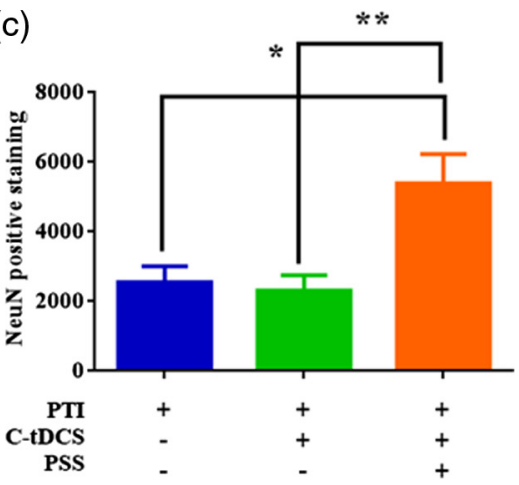

Fig. 6 Neuroprotective effects of C-tDCS + PSS. ( $a$ and b) NeuN staining (a) was used to visualize neurons in selected areas I and II, as shown in (b). The results showed that PTI markedly reduced the number of neurons compared with the contralateral side and that C-tDCS + PSS treatment protected neurons in the infarcted region following PTI. Scale bar $=250 \mu \mathrm{m}$. (c) Cell counts of NeuN-positive cells revealed significant preservation by more than 2 -fold in the C-tDCS + PSS + PTI group compared with the untreated group in the ischemic region $I . n=5$ to $6 .{ }^{*} p<0.05$ and ${ }^{* *} p<0.01$. 
group versus all other groups. A potential reason for this unexpected $\mathrm{SO}_{2}$ value might be due to the instant collateral circulation of the deeper microvessels of this batch of rats. ${ }^{44,45}$ Since the $880-\mathrm{nm}$ wavelength of light penetrates to a deeper tissue region, the effect of the potential deep collateral circulation could be observed by investigating $\mathrm{SO}_{2}$, while $\mathrm{CBV}$ $(808 \mathrm{~nm})$ could only examine the blood vessels at a surface region.

\subsection{Evaluations of Infarct Volume and Behavior}

The infarct volume obtained in rats treated with C-tDCS + PSS was significantly reduced by $50 \%$ and $40 \%$ compared with the no treatment group and the C-tDCS-treated group, respectively $(p<0.05)$. The PTI- and C-tDCS-only groups were not significantly different [Fig. 5(a)], showing that only C-tDCS + PSS is an effective intervention in reducing the infarct size following the ischemic insult. This result is also supported by our previous study, ${ }^{22}$ as we have shown that infarct volume can be reduced if PSS is delivered during the hyperacute phase of ischemia (i.e., $5 \%, 10 \%$, and $14 \%$ if applied immediately, 1 and $2 \mathrm{~h}$ postischemia, respectively; in this study, PSS was applied 30 min postischemia). ${ }^{22}$ Similarly, as shown in Fig. 5(b), the grip strength of the C-tDCS + PSS-treated group was well preserved and significantly stronger $(634 \pm 56 \mathrm{~g}$ force) than the grip strength of both the untreated $(495 \pm 39 \mathrm{~g})$ and the C-tDCS-treated $(534 \pm 68 \mathrm{~g})$ groups $(p<0.05)$. In addition, the grip strength of the C-tDCS + PSS group was also the closest to the baseline value of healthy animals $(783 \pm 79 \mathrm{~g})$. Thus, C-tDCS + PSS was able to induce the greatest restoration in the grip strength of the rats after ischemia.

\subsection{Neuroprotective Effect of Therapeutic Interventions}

NeuN immunohistochemical staining in the infarcted area was observed to be markedly decreased following PTI compared with the noninfarcted contralateral side (Fig. 6). Upon treatment, the number of NeuN-positive cells was markedly preserved (2-fold higher) in the C-tDCS + PSS-treated group compared with the no treatment group $(p<0.05)$. However, there was no significant difference in the C-tDCS-treated group compared with the PTI group.

In contrast, as shown in Fig. 7, ED-1-immunopositive cells significantly increased in the infarcted area following PTI in the untreated group $\left(2809 \pm 555\right.$ cells per $\left.\mathrm{mm}^{2}\right)$. Similar cell counts were obtained in the C-tDCS-treated group $\left(2739 \pm 307\right.$ cells per $\left.\mathrm{mm}^{2}\right)$, indicating microglia activation in both groups. In the C-tDCS + PSS-treated group, however, the obtained cell counts $\left(440 \pm 104\right.$ cells per $\left.\mathrm{mm}^{2}\right)$ were only $\sim 15 \%$ of those in the other groups, indicating a drastic suppression of microglial activation by C-tDCS + PSS treatment. The above results showed that the C-tDCS + PSS intervention may decrease infarct volume and improve functions such as grip strength by protecting against neuronal loss and suppressing inflammatory responses.

\section{Discussion}

Cortical spreading depression (CSD) is a spreading wave of electrical silence observed in stroke animal models and ischemia patients. ${ }^{46,47}$ It has been shown that CSD is associated with tissue hypoxia, an increase in oxygen demand exceeding the normal supply level, which may cause secondary neuronal (a)
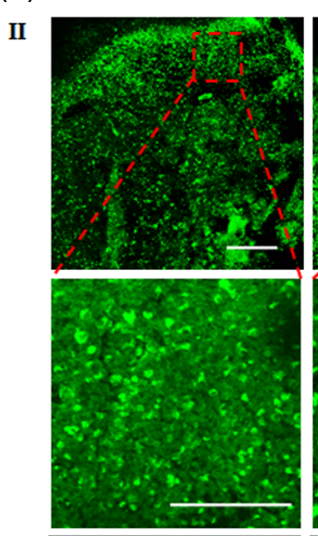

I

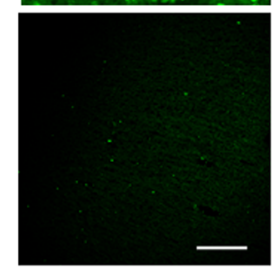

PTI + C-tDCS
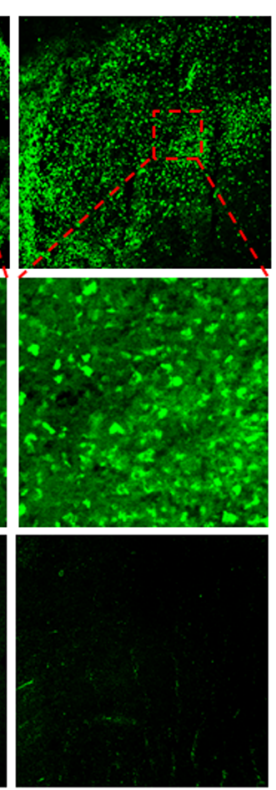

PTI + C-tDCS + PSS
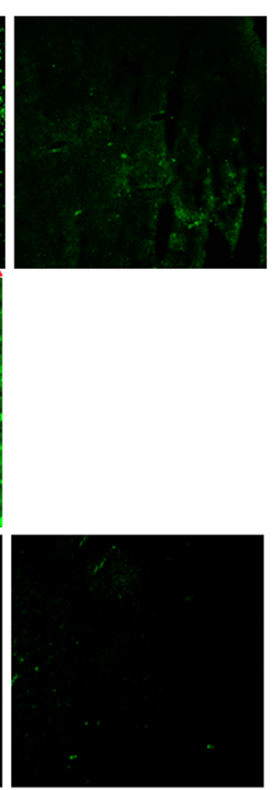

(b)

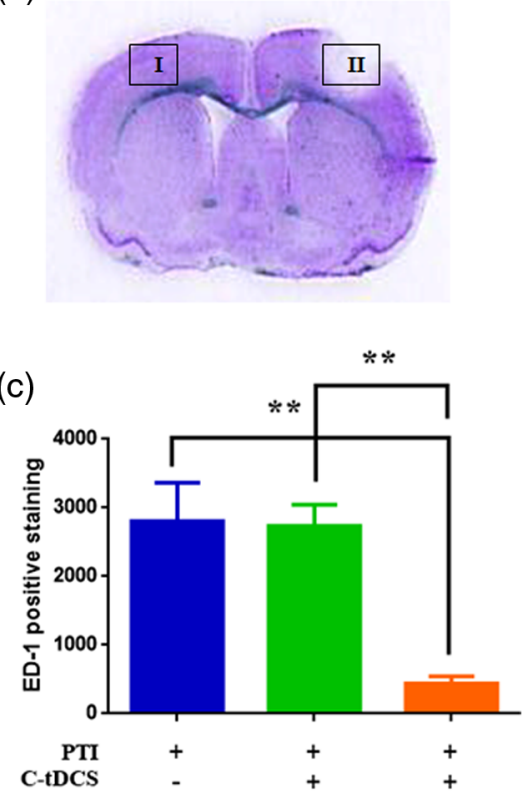

Fig. 7 C-tDCS + PSS suppressed microglia activation. (a and b) ED-1 staining was used to visualize activated microglia in selected areas I and II, as shown in (b). The results showed that PTI markedly increased the number of activated microglia compared with the contralateral side and that C-tDCS + PSS treatment prevented microglia activation in the infarcted region following PTI. a(II) lower panel represents the magnification of images taken from the respective ED-1 staining of infarcted regions, indicating the amoeboid reactive microglia. a(II) top panel scale bar $=250 \mu \mathrm{m}$. a(II) bottom panel scale bar $=125 \mu \mathrm{m}$. a(I) scale bar $=250 \mu \mathrm{m}$. (c) Cell counts of ED-1-positive cells showed a significant reduction by more than 2 -fold in the C-tDCS + PSS + PTI group in ischemic region $I . n=5$ to 6 . ${ }^{\star \star} p<0.01$. 
damage and contribute to lesion progression in the ischemic penumbra. ${ }^{46,47}$ In addition, researchers have also linked glutamate with CSD. ${ }^{14,47,48}$ Glutamate in the ischemic core may trigger CSD that propagates in the penumbra. ${ }^{14,48}$ In addition, glutamate excitotoxicity is one of the main mechanisms that damages nerve cells by excessive stimulation in the early phase of ischemic stroke. ${ }^{13,48} \mathrm{C}$-tDCS is a potential/noninvasive method able to inhibit CSD wave propagation because it can limit the abnormal release of glutamate and other excitatory neurotransmitters from damaged neurons after stroke. ${ }^{14}$ In other words, damage due to glutamate excitotoxicity postischemia is reduced by C-tDCS, salvaging dying neurons. ${ }^{49}$ Additionally, it has been shown that the number of slow potential changes (SPCs), which invariably accompany the transient depression of neural activity post-CSD, is related to infarct size. ${ }^{13,50,51}$ The number of SPCs in the hyperacute phase of ischemia also decreased when C-tDCS was applied in a timely manner in an animal ischemia model, reducing the infarct size. ${ }^{13}$ Therefore, global stimulation such as C-tDCS may be able to modulate the entire ischemic region via decreasing neurotransmitter release and causing fewer CSD/SPCs to prevent lesion expansion in the ischemic penumbra. ${ }^{20}$

However, C-tDCS has been found to afford only modest $(\sim 20 \%$ to $30 \%)$ reductions in infarct size in various stroke models. ${ }^{13,14}$ Wachter et al. ${ }^{19}$ reported that tDCS induces polarity-specific changes in cortical blood perfusion in rats. Specifically, A-tDCS is able to increase CBF, while C-tDCS leads to a decrease in CBF. In fact, reperfusion is an essential event for protecting the ischemic region after stroke by restoring blood flow to the damaged area, salvaging the tissues in the ischemic penumbra. ${ }^{22,27}$ Thus, although C-tDCS inhibits the release of glutamate and reduces CSD/SPCs, it somehow functions only passively to stop the expansion of exacerbation, instead of initiating active protection. Therefore, we argued that the effectiveness of C-tDCS could be markedly improved by integrating with a second complementary stimulation modality.

Previous studies have shown that many types of stimulation methods are able to modulate regional $\mathrm{CBF}$, including repetitive transcranial magnetic stimulation (rTMS), deep brain stimulation (DBS), pulsed transcranial ultrasound stimulation (pTUS), and PSS. ${ }^{22,52-55}$ Burnett et al. ${ }^{53}$ showed that electrical forepaw stimulation (i.e., PSS) is one method that is able to decrease infarct size because the PSS-induced blood flow increase exceeds the increase in metabolic demand after middle cerebral artery occlusion (MCAo), thus preventing the infarct. Our previous studies based on a rat PTI model also indicated that both unilateral and bilateral PSS can protect brain tissues in the ischemic penumbra through reperfusion. ${ }^{22,41}$ We found that focal stimulation in the form of PSS is able to induce reperfusion in the ischemic region during the hyperacute phase; therefore, we hypothesized that a combined treatment modality of C-tDCS + PSS should provide improved effectiveness compared with C-tDCS alone. The results obtained in the present study clearly demonstrated that C-tDCS and PSS, when sequentially applied to PTI-induced animals during the hyperacute phase, outperformed C-tDCS-only intervention based on all parameters measured.

We used the PPA of SSEPs, ADR, and DTABR as measures for neural activities to evaluate the effect of the interventions on preserving neural function in the hyperacute phase of ischemia. As shown in Fig. 3, PPA of SSEPs and ADR were decreased by PTI induction, which were reversed by both C-tDCS and C-tDCS + PSS treatments. However, the latter treatment may be considered superior because it restored the parameters to levels much closer to baseline values. As expected, DTABR was increased by PTI induction. This is consistent with previously reported increases after stroke due to increased proportions of slow waves, including the delta and theta bands. ${ }^{39,40}$ However, for this parameter, no differences were observed between the two treatment modalities, and the reason remains unknown. Additional investigations are needed to further elucidate these results and advance our understanding.

The hemodynamic parameters revealed that there were significant differences between the untreated group and both treated groups, as the $\mathrm{CBV}$ and $\mathrm{SO}_{2}$ values were increased by at least $20 \%$ in the treated groups at all three B-scan positions, indicating increased reperfusion. Although the CBV and $\mathrm{SO}_{2}$ values of the C-tDCS + PSS group were only slightly greater than those of the C-tDCS-only group ( $\sim 5 \%$ in CBV and $15 \%$ in $\mathrm{SO}_{2}$ ), these improvements in hemodynamics still contribute significantly to the protection against ischemic damages.

Neuroprotective effects can be elicited by mitigating neuronal cell death and inflammatory responses of immune cells. ${ }^{8,56-58}$ Glutamate excitotoxicity plays a major role in ischemic injuries, and neuroprotective approaches primarily focus on blocking excitotoxicity-induced neuronal death. ${ }^{1}$ PeruzzottiJametti et al. ${ }^{13}$ indicated that C-tDCS is able to decrease glutamate activity in the ischemic region by modulating NMDA receptors, while Stagg et al. ${ }^{14,18}$ observed a significant reduction in glutamate after C-tDCS due to a decreased rate of glutamate synthesis from glutamine with reduced excitatory neuronal transmission. However, the present results showed that CtDCS alone did not provide any significant protection against neuronal death (Fig. 6), nor did it reduce microglia activation (Fig. 7). Although C-tDCS is able to inhibit the release of glutamate, it also decreased blood flow in the ischemic region (Fig. 4). ${ }^{19,22}$ C-tDCS would, therefore, cause elevated potassium concentrations and then calcium influx, which exacerbates ischemic damage. ${ }^{22,25}$ Our results with respect to SSEPs and ADR showed that the C-tDCS-only treatment caused exaggerated recoveries to levels several times greater than the baseline level (Fig. 3), which is consistent with elevated potassium concentrations. According to a previous study, ${ }^{25}$ elevated $\mathrm{K}^{+}$ concentrations can invert neurovascular function to cause vasoconstriction and then further decrease blood supply to the ischemic region, inducing neuronal death. This may explain our observations that the C-tDCS-only treatment was ineffective. In stark contrast, the combined use of C-tDCS and PSS induced dramatic preservation of the number of neurons (Fig. 6) but decreased the number of active microglia (Fig. 7).

Immune responses can be triggered by elevated levels of reactive oxygen species (ROS) and neuronal necrosis in the presence of ischemia, ultimately leading to inflammatory cell activation and leukocytes infiltration. ${ }^{8,49,57}$ Therefore, any therapeutic interventions able to block various aspects of the inflammatory cascade may be useful for ameliorating injury due to ischemia. ${ }^{8,57,58}$ The inflammatory response may also be suppressed by regulating ROS. ROS stimulates ischemic cells/neurons to secrete inflammatory cytokines and chemokines, causing further release of a variety of cytotoxic agents, such as more cytokines/ROS, matrix metalloproteinases, and nitric oxide. ${ }^{57}$ These substances worsen ischemic damage, leading to brain 
edema, hemorrhage, and cell death. ${ }^{8,57}$ Thus, our observation that C-tDCS + PSS could abolish the activation of microglia to a large extent indicated a marked reduction in inflammatory responses and ROS generation. ${ }^{14,19}$ In addition, neural stem cell aggregation may be another cause of inhibition of inflammatory responses. ${ }^{59}$ Rueger et al. ${ }^{59,60}$ indicated that the C-tDCSinduced recruitment of proliferating neural stem cells was comparable to that induced pharmacologically, e.g., via activation of the Notch receptor, which suppressed inflammation in damaged tissue and removed debris in the peri-infarct tissue, improving neurological function.

We also compared the therapeutic efficacies of the integrated C-tDCS + PSS intervention and the stand-alone PSS treatments investigated in our previous studies. ${ }^{22,41}$ The neural activity results from previous studies showed that bilateral PSS with suitable intensity (i.e., $2 \mathrm{~mA}$ ) applied immediately after ischemia onset restores SSEPs and ADR closer to the baseline values but does not confer a complete recovery. ${ }^{41}$ However, our current study demonstrated that C-tDCS + PSS facilitated recovery of both parameters to levels slightly higher than the baseline level even at $4 \mathrm{~h}$ after ischemia onset. Therefore, these results suggest that C-tDCS + PSS might be a better intervention for restoring neural activity after ischemia compared with PSS alone.

On the other hand, the results of hemodynamics at the ischemic induction region indicated that the C-tDCS + PSS intervention resulted in much lesser restoration of $\mathrm{CBV}$ and $\mathrm{SO}_{2}$ (to $\sim 50 \%$ of the baseline values) compared with the stand-alone PSS intervention, which was able to restore hemodynamics to almost $70 \%$ of the baseline values following intervention. ${ }^{22,41}$ This difference could be attributed to the effect of C-tDCS, which decreases blood volume globally over the ischemic hemisphere. This implies that the ischemia induction location (i.e., the ischemic core) cannot be salvaged even when PSS is applied subsequently after C-tDCS. However, after $\mathrm{C}$-tDCS + PSS intervention, the $\mathrm{CBV}$ and $\mathrm{SO}_{2}$ values of the ischemic penumbra were restored (downstream) to levels even higher than the baseline values (upstream). That is, although the efficacy of C-tDCS + PSS in restoring hemodynamics at the ischemic core is worse than stand-alone PSS, the integrated intervention is still able to salvage the penumbra. Therefore, according to the above comparisons, we can infer that the neurovascular effect of C-tDCS + PSS is similar to PSS treatment (if applied immediately postischemia), indicating that C-tDCS + PSS may be a potential therapeutic intervention for restoring neurovascular function postischemia.

Moreover, the stand-alone PSS treatment was able to protect the ischemic region if administered immediately postischemia. ${ }^{22,41}$ However, PSS remains a double-edged sword, as treatment applied at $2 \mathrm{~h}$ postischemia may potentially exacerbate the stroke outcome. ${ }^{22}$ Thus, due to the constrained therapeutic time window of PSS, a clinical intervention (which is easier to perform during the hyperacute phase immediately poststroke) will be more suitable for integrating with PSS for translation to a potential treatment. This is another reason why we proposed/evaluated C-tDCS + PSS for the treatment of ischemia in this study. We further concluded that the enhanced effectiveness of this treatment is likely due to the inhibition of neurotransmitter release and CSD by C-tDCS and the additional focal stimulation. ${ }^{13,14,56}$ That is, PSS (or any stimulation method that can modulate regional hemodynamics) could actively recover the functions of both neural activity and hemodynamics (which might be compromised by C-tDCS) for stroke protection. This is the first time an integrated physical stimulation strategy has been utilized for salvaging the injured stroke area.

There are also some discrepancies between our current results and previous studies regarding the effect of C-tDCSonly treatment in ischemic stroke. ${ }^{13,14}$ Despite reporting a comparable infarct size, Peruzzotti-Jametti et al. ${ }^{14}$ observed that C-tDCS-treated animals displayed fewer ionized calcium-binding adapter molecule one cells in the ischemic region, indicating a decreased inflammatory response compared with the untreated group at $24 \mathrm{~h}$ post-MCAo. In addition, their results demonstrated that C-tDCS treatment preserved cortical neurons and promoted better clinical recovery compared with no treatment and A-tDCS treatment. This discrepancy might be a consequence of differences in experimental conditions, notably that a mouse MCAo model was used as opposed to the present rat PTI model. The MCAo model is also characterized by increased pathophysiology due to delayed reperfusion deficits. ${ }^{61}$

\section{Conclusions}

We propose a new integrated therapeutic intervention for protecting the ischemic area during the hyperacute phase of stroke. This study demonstrates that C-tDCS + PSS intervention is an effective method for facilitating recovery following PTI based on the strategy of coupled global + focal protection. Our results indicated that C-tDCS + PSS intervention outperformed $\mathrm{C}$-tDCS-only intervention and was able to restore neurovascular function, inhibit microglia activation, and protect neurons from ischemic injury. In addition, based on comparisons of the current results with our previous studies, we believe that the effects of the current integrated intervention are similar to PSS-only interventions but that the integrated intervention can be more easily applied in clinical studies due to the common use of tDCS devices. That is, the proposed therapeutic strategy could be smoothly translated to clinical trials by employing C-tDCS paired with any stimulation method that can modulate regional CBF, such as rTMS, DBS, pTUS, and PSS. In the future, we will evaluate more potential therapeutic interventions for ischemia and further utilize this ECoG-fPAM technology to probe neurovascular function in different disease animal models.

\section{Disclosures}

The authors declare no conflicts of interest.

\section{Acknowledgments}

This research was supported in part by the National University of Singapore through the Singapore Institute for Neurotechnology (SINAPSE) under grant numbers R-719003-100-305 and R-719-000-010-592. We also thank the Ministry of Science and Technology of Taiwan for financial support under grant number of 105-2221-E-400-006 and 106-2221E-400-004 and the National Health Research Institutes of Taiwan under grant numbers BN-106-PP-15, BN-107-PP-15, and BN-106-SP-02.

\section{References}

1. J. M. Lee, G. J. Zipfel, and D. W. Choi, "The changing landscape of ischaemic brain injury mechanisms," Nature 399, A7-A14 (1999).

2. S. N. Azizi et al., "Determination of epinephrine in pharmaceutical formulation by an optimized novel luminescence method using CdS quantum dots as sensitizer," J. Fluoresc. 23(2), 227-235 (2013). 
3. A. S. Go et al., "Heart disease and stroke statistics-2013 update: a report from the American Heart Association," Circulation 127(1), e6-e245 (2013).

4. S. H. Shon et al., "Predictors of hemorrhage volume after intravenous thrombolysis," J. Stroke Cerebrovasc. Dis. 25(10), 2543-2548 (2016).

5. R. Abu Fanne et al., "Blood-brain barrier permeability and tPAmediated neurotoxicity," Neuropharmacology 58(7), 972-980 (2010).

6. A. Majid, "Neuroprotection in stroke: past, present, and future," ISRN Neurol. 2014, 515716 (2014).

7. S. Roth and A. Liesz, "Stroke research at the crossroads-where are we heading?" Swiss Med. Wkly. 146, w14329 (2016).

8. S. J. Chan et al, "Neuroprotective effects of andrographolide in a rat model of permanent cerebral ischaemia," Br. J. Pharmacol. 161(3), 668-679 (2010).

9. F. C. Barone, "Ischemic stroke intervention requires mixed cellular protection of the penumbra," Curr. Opin. Invest. Drugs 10(3), 220-223 (2009).

10. M. Fisher et al, "Update of the stroke therapy academic industry roundtable preclinical recommendations," Stroke 40(6), 2244-2250 (2009).

11. M. A. Nitsche and W. Paulus, "Excitability changes induced in the human motor cortex by weak transcranial direct current stimulation," J. Physiol. 527(Pt 3), 633-639 (2000).

12. T. Jiang et al, "Effects of transcranial direct current stimulation on hemichannel pannexin-1 and neural plasticity in rat model of cerebral infarction," Neuroscience 226, 421-426 (2012).

13. F. Notturno et al, "Neuroprotective effect of cathodal transcranial direct current stimulation in a rat stroke model," J. Neurol. Sci. 342(1-2), 146151 (2014).

14. L. Peruzzotti-Jametti et al, "Safety and efficacy of transcranial direct current stimulation in acute experimental ischemic stroke," Stroke 44(11), 3166-3174 (2013).

15. F. Fregni and A. Pascual-Leone, "Technology insight: noninvasive brain stimulation in neurology-perspectives on the therapeutic potential of rTMS and tDCS," Nat. Clin. Practice Neurol. 3(7), 383-393 (2007).

16. M. A. Nitsche et al, "Transcranial direct current stimulation: state of the art 2008," Brain Stimul. 1(3), 206-223 (2008).

17. F. Hummel et al, "Effects of non-invasive cortical stimulation on skilled motor function in chronic stroke," Brain 128(Pt. 3), 490-499 (2005).

18. C. J. Stagg et al, "Polarity-sensitive modulation of cortical neurotransmitters by transcranial stimulation," J. Neurosci. 29(16), 5202-5206 (2009).

19. D. Wachter et al, "Transcranial direct current stimulation induces polarity-specific changes of cortical blood perfusion in the rat," Exp. Neurol. 227(2), 322-327 (2011).

20. T. Tanaka et al, "Transcranial direct-current stimulation increases extracellular dopamine levels in the rat striatum," Front. Syst. Neurosci. 7, 6 (2013).

21. L. D. Liao et al., "Transcranial imaging of functional cerebral hemodynamic changes in single blood vessels using in vivo photoacoustic microscopy," J. Cereb. Blood Flow Metab. 32(6), 938-951 (2012).

22. L. D. Liao et al., "Rescue of cortical neurovascular functions during the hyperacute phase of ischemia by peripheral sensory stimulation," Neurobiol. Dis. 75C, 53-63 (2015).

23. B. D. Watson et al., "Induction of reproducible brain infarction by photochemically initiated thrombosis," Ann. Neurol. 17(5), 497-504 (1985).

24. H. Nakase et al., "Use of local cerebral blood flow monitoring to predict brain damage after disturbance to the venous circulation: cortical vein occlusion model by photochemical dye," Neurosurgery 37(2), 280-286 (1995).

25. Y. H. Liu et al., "Assessment of neurovascular dynamics during transient ischemic attack by the novel integration of micro-electrocorticography electrode array with functional photoacoustic microscopy," Neurobiol. Dis. 82, 455-465 (2015).

26. L. D. Liao et al., "Imaging brain hemodynamic changes during rat forepaw electrical stimulation using functional photoacoustic microscopy," Neurolmage 52(2), 562-570 (2010).

27. R. D. Frostig, C. C. Lay, and M. F. Davis, "A rat's whiskers point the way toward a novel stimulus-dependent, protective stroke therapy," Neuroscientist 19(3), 313-328 (2013).

28. S. Roston, "The blood flow of the brain," Bull. Math. Biol. 29(3), 541548 (1967).
29. F. Fazekas et al., "Magnetic resonance imaging correlates of transient cerebral ischemic attacks," Stroke 27(4), 607-611 (1996).

30. M. A. Al-Rawi, F. B. Hamdan, and A. K. Abdul-Muttalib, "Somatosensory evoked potentials as a predictor for functional recovery of the upper limb in patients with stroke," J. Stroke Cerebrovasc. Dis. 18(4), 262-268 (2009).

31. P. Tzvetanov, R. T. Rousseff, and P. Atanassova, "Prognostic value of median and tibial somatosensory evoked potentials in acute stroke," Neurosci. Lett. 380(1-2), 99-104 (2005).

32. K. Sakatani, H. Iizuka, and W. Young, "Somatosensory evoked potentials in rat cerebral cortex before and after middle cerebral artery occlusion," Stroke 21(1), 124-132 (1990).

33. D. Wu et al, "Study of the origin of short- and long-latency SSEP during recovery from brain ischemia in a rat model," Neurosci. Lett. 485(3), 157-161 (2010).

34. S. J. Zhang et al, "EEG patterns from acute to chronic stroke phases in focal cerebral ischemic rats: correlations with functional recovery," Physiol. Meas. 34(4), 423-435 (2013).

35. J. Claassen et al, "Quantitative continuous EEG for detecting delayed cerebral ischemia in patients with poor-grade subarachnoid hemorrhage," Clin. Neurophysiol. 115(12), 2699-2710 (2004).

36. G. Assenza et al, "Neuronal functionality assessed by magnetoencephalography is related to oxidative stress system in acute ischemic stroke," NeuroImage 44(4), 1267-1273 (2009).

37. S. P. Finnigan et al, "Correlation of quantitative EEG in acute ischemic stroke with 30-day NIHSS score: comparison with diffusion and perfusion MRI," Stroke 35(4), 899-903 (2004).

38. C. Machado et al., "Assessing acute middle cerebral artery ischemic stroke by quantitative electric tomography," Clin. EEG Neurosci. 35(3), 116-124 (2004).

39. R. V. Sheorajpanday et al., "Additional value of quantitative EEG in acute anterior circulation syndrome of presumed ischemic origin," Clin. Neurophysiol. 121(10), 1719-1725 (2010).

40. R. V. Sheorajpanday et al., "Quantitative EEG in ischemic stroke: correlation with functional status after 6 months," Clin. Neurophysiol. 122(5), 874-883 (2011).

41. L. D. Liao et al., "Improving neurovascular outcomes with bilateral forepaw stimulation in a rat photothrombotic ischemic stroke model," Neurophotonics 1(1), 011007 (2014).

42. N. I. Wood et al., "Motor dysfunction in a photothrombotic focal ischaemia model," Behav. Brain Res. 78(2), 113-120 (1996).

43. H. Y. Lai et al., "Design, simulation and experimental validation of a novel flexible neural probe for deep brain stimulation and multichannel recording," J. Neural Eng. 9(3), 036001 (2012).

44. E. Cuccione et al., "Cerebral collateral circulation in experimental ischemic stroke," Exp. Transl. Stroke Med. 8, 2 (2016).

45. O. Y. Bang, M. Goyal, and D. S. Liebeskind, "Collateral circulation in ischemic stroke: assessment tools and therapeutic strategies," Stroke 46(11), 3302-3309 (2015).

46. T. Takano et al., "Cortical spreading depression causes and coincides with tissue hypoxia," Nat. Neurosci. 10(6), 754-762 (2007).

47. J. P. Dreier, "The role of spreading depression, spreading depolarization and spreading ischemia in neurological disease," Nat. Med. 17(4), 439447 (2011).

48. G. G. Somjen, "Mechanisms of spreading depression and hypoxic spreading depression-like depolarization," Physiol. Rev. 81(3), 10651096 (2001)

49. U. Dirnagl, C. Iadecola, and M. A. Moskowitz, "Pathobiology of ischaemic stroke: an integrated view," Trends Neurosci. 22(9), 391-397 (1999).

50. T. Back et al., "Induction of spreading depression in the ischemic hemisphere following experimental middle cerebral artery occlusion: effect on infarct morphology," J. Cereb. Blood Flow Metab. 16(2), 202-213 (1996).

51. C. Dohmen et al., "Spreading depolarizations occur in human ischemic stroke with high incidence," Ann. Neurol. 63(6), 720-728 (2008).

52. J. S. Perlmutter et al., "Blood flow responses to deep brain stimulation of thalamus," Neurology 58(9), 1388-1394 (2002).

53. M. G. Burnett et al., "Electrical forepaw stimulation during reversible forebrain ischemia decreases infarct volume," Stroke 37(5), 1327-1331 (2006).

54. A. M. Speer et al., "Intensity-dependent regional cerebral blood flow during $1-\mathrm{Hz}$ repetitive transcranial magnetic stimulation (rTMS) in 
healthy volunteers studied with $\mathrm{H} 215 \mathrm{O}$ positron emission tomography: II. Effects of prefrontal cortex rTMS," Biol. Psychiatry 54(8), 826-832 (2003).

55. T. Guo et al., "Pulsed transcranial ultrasound stimulation immediately after the ischemic brain injury is neuroprotective," IEEE Trans. Biomed. Eng. 62(10), 2352-2357 (2015).

56. C. A. McCarthy et al., "Angiotensin AT2 receptor stimulation causes neuroprotection in a conscious rat model of stroke," Stroke 40(4), 1482-1489 (2009).

57. Q. Wang, X. N. Tang, and M. A. Yenari, "The inflammatory response in stroke," J. Neuroimmunol. 184(1-2), 53-68 (2007).

58. G. Stoll, S. Jander, and M. Schroeter, "Inflammation and glial responses in ischemic brain lesions," Prog. Neurobiol. 56(2), 149-171 (1998).

59. M. A. Rueger et al., "Multi-session transcranial direct current stimulation (tDCS) elicits inflammatory and regenerative processes in the rat brain," PLoS One 7(8), e43776 (2012).

60. J. K. Ryu et al., "Neural progenitor cells attenuate inflammatory reactivity and neuronal loss in an animal model of inflamed $\mathrm{AD}$ brain," J. Neuroinflammation 6, 39 (2009).

61. F. E. Burrows et al., "Delayed reperfusion deficits after experimental stroke account for increased pathophysiology," J. Cereb. Blood Flow Metab. 35(2), 277-284 (2015).

Yu-Hang Liu is a PhD student in the Department of Electrical and Computer Engineering at National University of Singapore. He received his BS degree from National Central University, Taiwan, in electrical engineering (2008) and his MS degree from National Chiao Tung University, Taiwan, in biomedical engineering (2010). Photoacoustic imaging, brain optical imaging, neural signal processing, and embedded system design of biomedical application are his main research interests.

Su Jing Chan received her $\mathrm{PhD}$ in pharmacology from National University of Singapore and studied NF-kB, leukotriene, transulfuration, discovery of new molecular in apoptotic pathways and stem cell labeling/homing in stroke. She currently joined Harvard University as a research fellow under the supervision of Prof. Eng Lo, with research focusing on biomaterials in promotion of endogenous tissue regeneration, astrocytes signaling, extracellular mitochondria release, and development of biomaterials enabling astrocytes polarization in ischemic and hemorrhagic stroke.

Han-Chi Pan received his $\mathrm{PhD}$ in neuroscience from National Yang Ming University, Taiwan, in January 2015. He is currently a postdoctoral researcher in NanoNeurophotonics Lab at Institute of Biomedical Engineering and Nanomedicine in National Health Research Institutes, Taiwan. $\mathrm{He}$ is interested in neuroimaging, electrophysiology, molecular neuroscience, and brain stimulation. His long-term goal is to understand the disease mechanisms and solve the problems of neurological disorders.

Aishwarya Bandla is a research fellow in the Neurophotonics Group of the Singapore Institute for Neurotechnology (SINAPSE). She received her PhD in biomedical engineering from the National University of Singapore in 2017. Her doctoral research investigated non-drug, non-invasive therapeutic interventions for hyperacute ischemic stroke, and chemotherapy-induced peripheral neuropathy. Her research interests include exploring the translational aspects of neurovascular functions for diagnosis and treatment of central and peripheral neurological disorders.

Nicolas K. K. King is an associate consultant at the National Neuroscience Institute, Singapore. He graduated with a bachelor of medicine and surgery from the University of Sheffield, UK, and obtained his PhD from Imperial College London, UK, where he investigated the modulation of cortical plasticity. He was awarded his specialist degree in neurosurgery (FRCS) after completing his neurosurgical training at the National Neuroscience Institute, Singapore.

Peter Tsun Hon Wong, BSc (Hons.) PhD, is a professor and former head of pharmacology, Yong Loo Lin School of Medicine, National University of Singapore. He is an associate editor of Neurochemistry International and Journal of Pharmacological Sciences and a council member of the International Society for Neurochemistry. His primary research interest is in stroke with a focus on novel therapeutic approaches. He has published over 130 articles in peer-reviewed journals with an h-index of 30 .

You-Yin Chen received his $\mathrm{PhD}$ in electrical engineering from the National Taiwan University, Taiwan, in 2004. Currently, he is a professor at the Department of Biomedical Engineering of the National Yang Ming University in Taipei, Taiwan. His research interests include the development of the multifunctional neuro-interface for brainmachine interface and deep brain stimulation in neurodegenerative disease, which operates at the crossroads between basic neural engineering, neurophysiology, and clinical care.

Wai Hoe $\mathbf{~ N g}$ is the medical director of the National Neuroscience Institute and academic chair of the SingHealth Duke-NUS Neuroscience Academic Neuroscience Programme. He is a neurosurgeon with subspecialty interests in neurosurgical oncology and stereotactic and functional neurosurgery.

Nitish V. Thakor is a professor of BME at Johns Hopkins and the director of Singapore Institute for Neurotechnology (SINAPSE) at National University of Singapore. His expertise is in the field of neurotechnology and medical instrumentation. $\mathrm{He}$ is a fellow of American Institute of Medical, Biological Engineering, IEEE, a founding fellow of BMES, and a fellow of the International Federation of Medical, Biological Engineering, and editor in chief of Medical and Biological Engineering and Computing.

Lun-De Liao received his $\mathrm{PhD}$ in electrical engineering from National Chiao Tung University, Taiwan, in 2012. He was a research scientist in Singapore Institute for Neurotechnology at National University of Singapore from August 2012-May 2014, and a senior research scientist from 2014-2016. In November 2015, he joined the Institute of Biomedical Engineering and Nanomedicine, National Health Research Institutes, Taiwan, as a principal investigator leading the NanoNeurophotonics Lab. He has published over 70 peer-reviewed $\mathrm{SCl}$ journal papers and holds 12 issued patents. He is interested in neurophotonics, experimental neuroscience, and optical microscopy. 